\title{
REMARKS ON A PROBLEM OF OBREANU
}

P. Erdơs and A. Rényi

(received December 15, 1962)

Let $a_{1}<a_{2}<\ldots$ be any sequence of integers. Assume that the infinite sequence of numbers $u_{n}$ satisfies the following condition: To every $\varepsilon>0$ there is an $n_{0}=n_{0}(\varepsilon)$ such that for all $\mathrm{n}>\mathrm{n}_{\mathrm{o}}$ and all $\mathrm{k}$

(1) $\quad\left|u_{n+a_{k}}-u_{n}\right|<\varepsilon$.

Obreanu asked (Problem P. 35 Can. Math. Bull.) under what conditions on the sequence $a_{1}<a_{2}<\ldots$ does (1) imply that the sequence $u_{n}$ is convergent. N. G. de Bruijn and P. Erdors proved that a necessary and sufficient condition for (1) to imply the convergence of $u_{n}$ is that the sequence $\left\{a_{n}\right\}$ be infinite and that the greatest common divisor of the $a_{n}$ should be 1 .

The condition (1) is very strong and is "nearly equivalent" to Cauchy's criterion for convergence. We discuss various conditions which are weaker than (1).

Assume first that the sequence $u_{n}$ satisfies

(2) $\lim _{n \rightarrow \infty} \overline{\lim }\left|u_{n+a}-u_{n}\right|=0$.

Condition (2) means that to every $\varepsilon>0$ there exists $n_{0}=n_{0}(\varepsilon)$ such that for $n>n_{0}$ we have $\left|u_{n+a}-u_{n}\right|<\varepsilon$ except for

Canad. Math. Bull. vol. 6, no. 2, May 1963. 
finitely many $r$ (the number of exceptional $r$ may of course depend on $n$ ). Denoting the sequence $a_{1}<a_{2}<\ldots$ by $A$ we shall prove

THEOREM 1 (2) implies the convergence of $\left\{u_{n}\right\}$ if and only if $\mathrm{A}$ satisfies the following two conditions:

(I) to every integer $d>1$ there are infinitely many $\mathrm{k}$ with $a_{k} \neq 0(\bmod d)$,

(II) $a_{k+1}-a_{k}$ does not tend to infinity as $k \rightarrow \infty$.

First we prove that (I) and (II) are necessary. This is clear for (I) since if (I) is not satisfied for a certain $d>1$ then the sequence $u_{n}$ with

$$
u_{n}=0 \text { if } n \equiv 0(\bmod d) \text { and } u_{n}=1 \text { otherwise, }
$$

clearly satisfies (2) and does not converge.

Next we show that (II) is necessary. Suppose A does not satisfy (II), i.e. $a_{k+1}-a_{k} \rightarrow \infty$ as $k \rightarrow \infty$. Put

$$
n=a_{i_{1}}+a_{i_{2}}+\ldots+a_{i_{r}}+\ell
$$

where $a_{i_{1}}$ is the greatest a not exceeding $n, a_{i_{2}}$ the greatest a not exceeding $n-a_{i_{1}}$, or $a_{i_{r}}$ is the greatest a not exceeding $n-\left(a_{i_{1}}+\ldots+a_{i_{r-1}}\right)$, and $0 \leq \ell<a_{1}$ (thus if $a_{1}=1, \ell$ is always 0 ). Put

$$
u_{n}=0 \text { if } i_{r}=1 \text { and } u_{n}=1 \text { if } i_{r} \neq 1
$$

e.g. if ( $i>i_{0}$ ) $n=a_{i}+a_{1}$ then $u_{n}=0$, while if $n=a_{i}+a_{2}$ then $u_{n}=1$. Thus $u_{n}$ is infinitely often 0 and infinitely 
often 1 and hence does not converge. On the other hand it is easy to see that the sequence (3) satisfies (2) since from $a_{k+1}-a_{k} \rightarrow \infty$ we obtain that $a_{k+1}-a_{k}>n$ for $k>k_{0}(n)$ and hence for these $k$ we have from (3) $u_{n+a_{k}}-u_{n}=0$, so that (2) is satisfied. This shows that our conditions are necessary.

Next we show that our conditions are sufficient, in other words we shall show that if $A$ satisfies (I) and (II) and the infinite sequence $\left\{u_{n}\right\}$ satisfies (2), then $\left\{u_{n}\right\}$ converges.

Since (II) is satisfied, there is a $\mathrm{T}$ for which

(4) $a_{k+1}-a_{k}=T$

has infinitely many solutions. First we show that for every i

(5) $\quad \lim _{\ell \rightarrow \infty}\left(u_{i+(\ell+1) T}-u_{i+l T}\right)=0$.

Let $\varepsilon>0$ be given; to prove (5) we shall show that for all $\ell>\ell_{0}(\varepsilon)$

(6) $\left|u_{i+(l+1) T}-u_{i+l} T\right|<\varepsilon$.

From (2) it follows that for sufficiently large fixed $\ell(\ell=\ell(\varepsilon))$ and every $r>r_{0}(\varepsilon, \ell)$

(7)

$$
\begin{aligned}
& \left|u_{i+l T+a_{r}}-u_{i+l T}\right|<\varepsilon / 2 \text { and } \\
& \left|u_{i+(l+1) T+a_{r}}-u_{i+(l+1) T}\right|<\varepsilon / 2 .
\end{aligned}
$$

Since (4) has infinitely many solutions there is a $k$ (in fact infinitely many such $k$ ) for which $a_{k+1}-a_{k}=T, k>r_{0}(\varepsilon, \ell)$.

Thus from (7) 
(8) $\quad\left|u_{i+l} T+a_{k+1}-u_{i+l ~ T}\right|<\varepsilon / 2$ and

$$
\left|u_{i+(\ell+1) T+a_{k}}-u_{i+(\ell+1) T}\right|<\varepsilon / 2 \text {. }
$$

(6) follows from (8) by subtraction ( since $i+(\ell+1) \mathrm{T}+\mathrm{a}_{\mathrm{k}}=$ $\left.i+\ell+a_{k+1}\right)$ (6) implies that for every $s$ and $i$

(9) $\quad \lim _{\ell \rightarrow \infty}\left(u_{i+(l+s) T}-u_{i+l} T\right)=0$.

From (9) we shall now deduce that for every fixed i

(10) $\lim _{\ell \rightarrow \infty} u_{i+\ell} T$

exists. If (10) did not exist there would exist an infinite sequence of integers $\xi_{j}, \lambda_{j}$ satisfying

(11) $\xi_{j} \equiv \lambda_{j} \equiv i(\bmod T), \quad \xi_{1}<\xi_{2}<\ldots, \xi_{j}<\lambda_{j}$

and

(12) $\left|u_{\xi_{j}}-u_{\lambda}\right|>c$

for a certain positive absolute constant c. From (2) we obtain that for sufficiently large $j$ and $r$

(13) $\left|u_{\xi_{j}+a}-u_{\xi_{j}}\right|<c / 4$ and $\left|u_{\lambda_{j}+a_{r}}-u_{\lambda_{j}}\right|<c / 4$.

From the first part of (11) we have $\xi_{j}-\lambda_{j}=s T$, and so from

(9) we have for sufficiently large $r$

$$
\left|u_{\xi_{j}+a}-u_{\lambda}+a_{j}\right|<c / 4\left(\xi_{j}+a_{r}=i+l T \text { of }(9)\right)
$$

(13) and (14) imply $\left|u_{\xi_{j}}-u_{\lambda}\right|<3 c / 4$ which contradicts (12), 
and hence (10) is proved.

If the limit in (10) does not depend on $i$ then $\left\{u_{n}\right\}$ converges and our theorem is proved. Assume thus that for two values $i_{1} \neq i_{2}(\bmod T)$

(15) $\lim _{\ell \rightarrow \infty} u_{i_{1}+\ell T}=\alpha_{1}, \lim _{\ell \rightarrow \infty} u_{i_{2}+\ell T}=\alpha_{2}, \alpha_{1}<\alpha_{2}$.

Choose $\varepsilon<\left(\alpha_{2}-\alpha_{1}\right) / 2 \mathrm{~T}^{2}$ and let $\ell$ be so large that for all $\mathrm{n}>\ell \mathrm{T}$ and all $\mathrm{r}$ except possibly for finitely many exceptions

(16) $\left|u_{n+a}-u_{n}\right|<\varepsilon$,

and choose $\ell_{0}$ so large that for every $\ell>l_{0} \ell_{1}>l_{0}$

$$
\left|u_{i_{1}+l} T-u_{i_{2}+l} T\right|>\left(\alpha_{2}-\alpha_{1}\right) / 2
$$

Denote by $j_{1}, \ldots, j_{r}$ those residue classes (mod $T$ ) for which the congruence $a_{n} \equiv j_{s}(\bmod T)$ has infinitely many solutions. By (I), $\left(j_{1}, j_{2}, \ldots, j_{r}, T\right)=1$ and therefore the congruence

$$
\sum_{s=1}^{r} x_{s} j_{s} \equiv i_{2}-i_{1}(\bmod T), \quad 0 \leq X_{s}<T
$$

is solvable (in fact every residue class ( $\bmod T$ ) can be represented in the form (18). We can find arbitrarily large $a^{\prime} s$ satisfying $\left(a_{n} \equiv j_{s}(\bmod T)\right.$ has infinitely many solutions)

$$
\mathrm{a}_{\mathrm{m}} \equiv \mathrm{j}_{\mathrm{s}}(\bmod \mathrm{T}) \quad 1 \leq \mathrm{s} \leq \mathrm{r}
$$

Put

$$
v=i+l T+\sum_{s=1}^{r} x_{s} a_{m_{s}}=i+l T+\sum_{j=1}^{y} b_{j}, y=\underset{s=1}{\sum} x_{s}<T^{2} \text { (by }
$$


where $X_{s}$ of the $b^{\prime} s$ are equal to $a_{m_{s}} \cdot$ From (19) and (18) we have

(20) $v=i_{2}+\ell_{1} T, \quad l{ }_{1} \geq \ell$.

We evidently have by (19), (as in the proof of Problem 35)

$$
\begin{aligned}
& \left|u_{v}-u_{i_{1}}+l T\right| \leq\left|u_{i_{1}}+\ell T+b_{1}-u_{i_{1}}+\ell T\right| \\
& +\left|u_{i_{1}}+l T+b_{1}+b_{2}-u_{i_{1}+l T+b_{1}}\right|+\ldots \\
& +\left|u_{i_{1}}+l T+\sum_{j=1}^{r} b_{j}-u_{i_{1}}+l T+\sum_{j=1}^{r-1} b_{j}\right|
\end{aligned}
$$

Now since each $b$ is an a, we have from (16) and (17) that for sufficiently large $\ell$ and sufficiently large $b^{\prime} s$ each summand at the right side of (21) is less than $\varepsilon$. Thus from (20), (21) and the definition of $\varepsilon$ we obtain by the last inequality of (19)

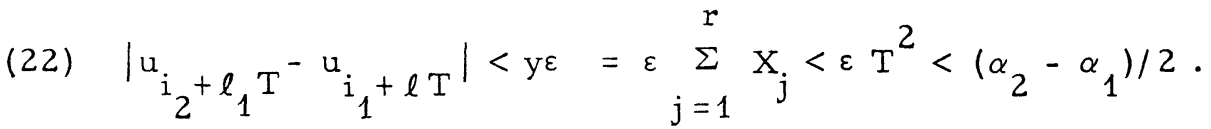

(22) contradicts (17) and this contradiction proves the convergence of $\left\{u_{n}\right\}$ and hence the proof of our theorem is complete.

We also considered the following modification of (2):

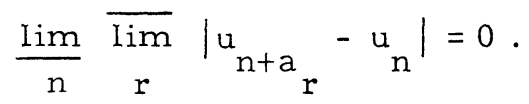

We proved

THEOREM 2 (23) implies the convergence of $\left\{u_{n}\right\}$ if and only if for every infinite sequence of integers $b_{1}<b_{2}<\ldots$ there is a $t$ such that the sequence 
(24) $\left\{a_{r}+b_{i}\right\} \quad 1 \leq r<\infty, \quad 1 \leq i \leq t$

contains all but a finite number of the integers $1,2, \ldots$

We suppress the proof of Theorem 2. It is easy to see that (24) is equivalent to the following condition which is perhaps more manageable: Let $b_{1}<b_{2}<\ldots$ be any infinite sequence of integers; then all but a finite number of the natural numbers are of the form $\left(a_{i}+b_{j}\right)$ where $i$ and $j$ are natural numbers.

Assume that we modify (2) as follows: To every $\varepsilon>0$ there exists an $n_{0}$ such that for $n>n_{0}$ we have $\left|u_{n+a_{k}}-u_{n}\right|<\varepsilon$ except for at most $t_{\varepsilon}$ values of $k$ where $t_{\varepsilon}$ depends only on $\varepsilon$ and not on $n$. We do not know what is the necessary and sufficient condition on the sequence $\left\{a_{k}\right\}$ that this should imply that $\left\{u_{n}\right\}$ converges.

University College London

England 